\title{
A GENERALIZED SKEWED EXPONENTIAL DISTRIBUTION WITH APPLICATION TO UNEMPLOYMENT AGE DISTRIBUTION
}

\author{
Seng Huat Ong'a, Wooi Chen Khoo ${ }^{2 b^{*}}$ \\ aDepartment of Actuarial Science and Applied Statistics, UCSI University, 56000 Kuala Lumpur, MALAYSIA. \\ Email: ongsh@um.edu.my ${ }^{1}$ \\ bepartment of Applied Statistics, School of Mathematical Sciences, Sunway University, 47500 Selangor, \\ MALAYSIA. Email: wooichenk@sunway.edu.my² \\ *Corresponding author: wooichenk@sunway.edu.my \\ Received: $22^{\text {nd }}$ Jul $2019 \quad$ Accepted: $11^{\text {th }}$ Oct 2019 \\ Published: $31^{\text {st }}$ Dec 2019 \\ DOI: https://doi.org/10.22452/mjs.vol38no3.2
}

\begin{abstract}
This paper introduces a generalization of the weighted or skewed exponential distribution. The motivation to consider this generalization stems from the interest in modelling unemployment data. The generalized skewed exponential distribution is shown to be log-concave, and as a consequence, a number of properties of the distribution are obtained. The generalized skewed exponential distribution is shown to give much better fit than the skewed exponential, Weibull, lognormal and logistic distributions for the unemployment age data.
\end{abstract}

Keywords: Empirical Modelling, Log-concavity, Transformation, Unimodality.

\section{INTRODUCTION}

The recent statistical literature is flooded with many extensions and generalizations of continuous statistical distributions. A variety of methods have been proposed to extent families of distributions by including extra parameters for greater flexibility, and also for a better fit in empirical modelling. For instance, Gupta and Kundu (1999) considered exponentiation of the exponential distribution, and Marshall and Olkin (1997) added one parameter to the survival function to create a wide family of distributions. The T-X family of distributions proposed by Alzaatreh, Lee and Famoye (2013) generates a broad range of statistical distributions. Although these families of distributions are mathematically interesting, many lack stochastic interpretations which are of concern to stochastic modellers and statistical data analysts.

One method of introducing an extra parameter has been considered by Azzalini (1985) in his derivation of the skew normal distribution. A random variable $X$ is said to have a skew normal distribution if its probability density function (pdf) is given by:

$$
f(x)=2 \phi(x) \Phi(\lambda x)
$$

where $\lambda$ is the skewness parameter, $\phi(x)$ and $\Phi(x)$ are the standard normal pdf and cumulative distribution function (cdf) respectively. Obviously this technique of introducing a skewness parameter is applicable to any continuous distribution. Gupta and Kundu (2009) derived the weighted exponential or skewed exponential distribution by introducing a skewness parameter following the method of Azzalini (1985). Skewed distributions 
are still attracting the attention of many researchers. Recently, Lee and McLachlan (2016) considered unification of the restricted and unrestricted skew t-mixture models. Jamalizadeh and Lin (2017) introduced scale-shape mixtures of skewnormal distributions as alternative models for asymmetric data in a wide variety of settings. Lin et al (2018) introduced a robust extension of the mixture of factor analysis models based on the restricted multivariate skew-t distribution, known as mixtures of skew-t factor analysis.

This paper proposes a generalization of the skewed exponential distribution of Gupta and Kundu (2009). The motivation for introducing the generalized skewed exponential distribution is due to the modelling of unemployment age data, especially youth unemployment. In many countries unemployment age distributions have a similarity which is a high proportion of youth unemployment. We have considered the unemployment data from Malaysia. The unemployment rate of Malaysia is relatively stable and low at around three percent which implies that the country is experiencing almost full employment. However, among this three percent of unemployed, the majority are young people. Youth unemployment is an important issue and most countries share the common characteristic of having a high youth unemployment relative to other age groups. Ochsen (2008) has examined the effect of age distribution on the unemployment rate. Ochsen (2008) considered data from OECD countries and showed that the distribution of unemployment by age has a hump-shape effect on the unemployment rate.

The generalized skewed exponential distribution is derived by exponentiating the skewed exponential distribution function, that is, by raising the cdf to a power. This is the method used by Gupta and Kundu (1999) to obtain their exponentiated exponential distribution. The organization of the paper is as follows. Section 2 defines the skewed exponential distribution and gives some properties. The generalization of the skewed exponential distribution is introduced in section 3. It is shown that the generalized skewed exponential distribution is log-concave. Section 4 considers parameter estimation by maximum likelihood for the skewed exponential distribution where the score functions have been derived. The fit to the age distribution for unemployment in Malaysia is also presented. The fit is compared with the skewed exponential, Weibull, lognormal and logistic distributions, and it is shown that the generalized skewed exponential distribution provides a superior fit. The conclusion is given in section 5 .

\section{SKEWED EXPONENTIAL DISTRIBUTION AND A GENERALIZATION}

\subsection{Skewed Exponential Distribution}

The weighted or skewed exponential distribution of Gupta and Kundu (2009) has pdf

$$
g(x)=\frac{\lambda+1}{\lambda} \alpha e^{-\alpha x}\left(1-e^{-\alpha \lambda x}\right), \quad \alpha, \lambda, x>0
$$

where $\alpha$ and $\lambda$ denote the scale parameter and skewness parameter respectively. The pdf of the skewed exponential distribution has shape which is very similar to the shape of the Weibull, gamma or generalized exponential distributions. This means that this distribution can be an alternative model for these distributions. The skewed exponential cdf is 


$$
G(x)=1-\frac{1}{\lambda}[(1+\lambda) \exp (-\alpha x)-\exp (-\alpha(1+\lambda) x)]
$$

The survival function and hazard function are respectively

$$
S(x)=1-G(x)=\frac{1}{\lambda}[(1+\lambda) \exp (-\alpha x)-\exp (-\alpha(1+\lambda) x)]
$$

and

$$
h(x)=\frac{g(x)}{S(x)}=\frac{\alpha(1+\lambda)(1-\exp (-\lambda \alpha x))}{(1+\lambda)-\exp (-\lambda \alpha x)}
$$

The properties of these functions and the log-concavity of the skewed exponential distribution are further elaborated next. Log-concavity of the skewed exponential distribution is stated in Gupta and Kundu (2009) but detailed proof is not given. We provide a proof of log-concavity in order to facilitate discussion about the log- concavity of the generalized skewed exponential distribution.

\subsection{Moments and Other Properties}

The moment generating function of the skewed exponential distribution is given by

$$
M_{X}(t)=\frac{\alpha^{2}(1+\lambda)}{t^{2}-2 \alpha t-\lambda \alpha t+\alpha^{2}+\lambda \alpha^{2}},-1<t<1
$$

We get the mean and the variance as

$$
\begin{gathered}
E[X]=\frac{2+\lambda}{\alpha(1+\lambda)} \\
\operatorname{var}(X)=E\left[X^{2}\right]-(E[X])^{2}=\frac{\lambda^{2}+2 \lambda+2}{\alpha^{2}(1+\lambda)^{2}}
\end{gathered}
$$

We next discuss the log-concavity of the skewed exponential distribution. A function $f(x)$ is said to be log-concave if $\ln f(x)$ is concave and log-convex if $\ln f(x)$ is a convex function. The $\log$ concavity or log-convexity of pdf's has many interesting implications regarding the properties of the distributions. This logconcavity property has many applications in life sciences, commerce, economics, political science, and industry (Bagnoli and
Bergstrom, 2005). The log-concavity of a function $f(x)$ on an interval $(a, b)$ is equivalent to $f^{\prime}(x) / f(x)$ being monotonically decreasing in $(a, b)$ or $(\ln f(x))^{\prime \prime}<0$ (Definition 2, Borzadaran and Borzadaran, 2011). We use this fact to show the log-concavity of the skewed exponential pdf. The logarithm of the skewed exponential pdf is given by 


$$
\ell(x)=\ln g(x)=\ln ((\lambda+1) \alpha / \lambda)+(-\alpha x)+\ln \left(1-e^{-\alpha \lambda x}\right) .
$$

Then

$$
\ell^{\prime}(x)=g^{\prime}(x) / g(x)=-\alpha+\alpha \lambda e^{-\alpha \lambda x} /\left(1-e^{-\alpha \lambda x}\right) .
$$

Observe that $\left(1-e^{-\alpha \lambda x}\right)$ is monotonically increasing since $\alpha \lambda e^{-\alpha \lambda x}$ is monotonically decreasing, and $\ell^{\prime}(x)$ is monotonically decreasing in $x$. Thus the skewed exponential pdf is log-concave, that is, the pdf is a Polya frequency density of order 2. Many properties, especially reliability properties, of the skewed exponential distribution follow from this log-concavity property (Borzadaran and Borzadaran, 2011, pages 205-206). For example,

(1) Skewed exponential distribution is strongly unimodal;

(2) Hazard function of the skewed exponential distribution is a non-decreasing function in $x$;
(3) Distribution function and survival function of the skewed exponential distribution are log-concave.

Log-concavity confers desirable statistical properties for estimators (see Balabdaoui, Rufibach and Wellner, 2009). For example, the nonparametric MLE of a log-concave density exists, is unique and has desirable consistency and rates of convergence properties. The exponential distribution is connected to many important distributions through transformation of random variables. As an example, if $X \sim \operatorname{Exponential}(\alpha)$, then it is found that $e^{-X} \sim \operatorname{Beta}(\alpha, 1)$, where the beta random variable $\operatorname{Beta}(\alpha, \beta)$ with pdf

$$
f(x)=\frac{x^{\alpha-1}(1-x)^{\beta-1}}{B(\alpha, \beta)}, 0<x<1 .
$$

Transformations of skewed exponential random variable are easily determined from those for the exponential distribution if we rewrite the pdf (2.4) as

$$
g(x)=\frac{\lambda+1}{\lambda} \alpha e^{-\alpha x}\left(1-e^{-\alpha \lambda x}\right)=\left(1+\frac{1}{\lambda}\right) \alpha e^{-\alpha x}+\left(-\frac{1}{\lambda}\right) \alpha(1+\lambda) e^{-\alpha(1+\lambda) x}
$$

$\alpha, \lambda, x>0$, that is, the sum of two exponential pdf's with parameters $\alpha$ and $\alpha(1+\lambda)$ respectively. To illustrate, let
$X \sim \operatorname{Skewed\operatorname {Exp}}(\alpha, \lambda)$, with pdf (2.3). The random variable $e^{-X}$ has pdf given by

$$
g(x)=\left(1+\frac{1}{\lambda}\right) f_{1}(x ; \alpha)+\left(-\frac{1}{\lambda}\right) f_{2}(x ; \alpha, \lambda)
$$

where $f_{1}(x ; \alpha)$ and $f_{2}(x ; \alpha, \lambda)$ are beta pdf's for $\operatorname{Beta}(\alpha, 1)$ and $\operatorname{Beta}(\alpha(1+\lambda), 1)$. 


\section{GENERALIZED SKEWED EXPONENTIAL DISTRIBUTION}

Following the idea of Gupta and Kundu (1999), a generalization of the skewed exponential distribution is obtained by raising the skewed exponential distribution function (2.2) to the power of $\beta$. This results in the generalized skewed exponential distribution function:

$$
F(x)=\left(1-\frac{1}{\lambda}[(1+\lambda) \exp (-\alpha x)-\exp (-\alpha(1+\lambda) x)]\right)^{\beta}
$$

The generalized skewed exponential distribution has pdf

$$
\begin{aligned}
f(x) & =F^{\prime}(x) \\
& =\beta \frac{(1+\lambda)}{\lambda} \alpha \exp (-\alpha x)(1-\exp (-\alpha \lambda x)) \\
& \cdot\left(1-\frac{1}{\lambda}[(1+\lambda) \exp (-\alpha x)-\exp (-\alpha(1+\lambda) x)]\right)^{\beta-1}
\end{aligned}
$$

Some plots of the pdf are given below in Figure 3.1. Note that as $\lambda$ decreases the skewness of the distribution increases.

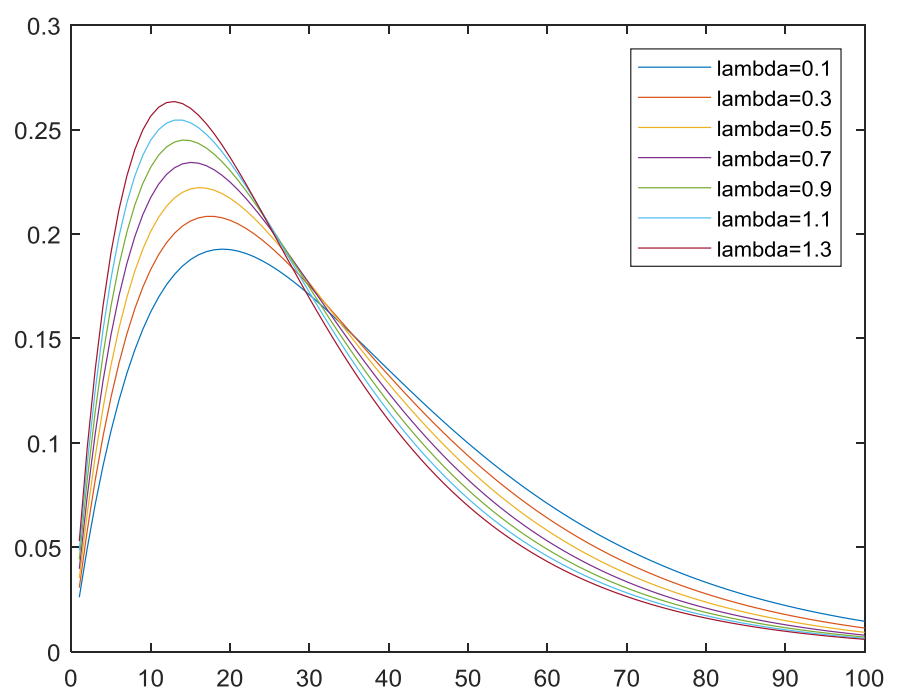

Figure 3.1. Plots of generalized skewed exponential pdf: beta $=1$, alpha $=0.5$, varying lambda

\subsection{Moment generating function}

The Laplace transform of the generalized skewed exponential pdf is given by

$$
L_{X}(s)=\int_{0}^{\infty} e^{-s t}\left(F(t)^{\beta}\right)^{\prime} d t=s T_{X}(s)
$$


where

$$
T_{X}(s)=\int_{0}^{\infty} e^{-s t} F(t)^{\beta} d t
$$

By using binomial expansion, it is found that

$$
T_{X}(s)=\sum_{k=0}^{\infty} \sum_{i=0}^{k} \frac{(\beta)_{k}}{k !} \lambda^{-k}(-1)^{i}\left(\begin{array}{l}
k \\
i
\end{array}\right) \frac{(1+\lambda)^{k-i}}{(\alpha(k+\lambda i)+s)} .
$$

Hence the moment generating function is given by

$$
M_{X}(t)=L_{X}(-s)=-s T_{X}(-s) .
$$

That is,

$$
M_{X}(t)=\sum_{k=0}^{\infty} \sum_{i=0}^{k} \frac{(\beta)_{k}}{k !} \lambda^{-k}(-1)^{i+1}\left(\begin{array}{l}
k \\
i
\end{array}\right) \frac{t(1+\lambda)^{k-i}}{(\alpha(k+\lambda i)-t)}
$$

From equation (3.2), the moments for the generalized skewed exponential distribution can be derived.

\subsection{Log-concavity}

We now show that the generalized skewed exponential distribution is log-concave. simplify the notation, let the cdf be written as

$$
F(x)=H(x)^{\beta}
$$

where

$$
H(x)=\left(1-\frac{1}{\lambda}[(1+\lambda) \exp (-\alpha x)-\exp (-\alpha(1+\lambda) x)]\right)
$$

with corresponding pdf $h(x)$. Pdf (3.1) becomes

$$
f(x)=\beta h(x) H(x)^{\beta-1} .
$$

As in the proof of log-concavity of the skewed exponential distribution, consider

$$
\ell(x)=\ln f(x)
$$


By using (3.3), we get

$$
\ell^{\prime}(x)=f^{\prime}(x) / f(x)=h^{\prime}(x) / h(x)+(\beta-1) h(x) / H(x) .
$$

Since the skewed exponential pdf $h(x)$ is log-concave, $h^{\prime}(x) / h(x)$ is monotonically decreasing with monotonically increasing hazard rate $h(x) /[1-H(x)]$. Then $h(x) / H(x)$ is monotonically decreasing. For $\beta \geq 1 \quad$ we conclude that $\ell^{\prime}(x)=f^{\prime}(x) / f(x)$ is monotonically decreasing and the generalized skewed exponential distribution is log-concave. This result is true for any distribution with cdf of the form $F(x)=H(x)^{\beta}$, where $H(x)$ is the base cdf, and is summarized in the following theorem.

Theorem: Let a distribution with pdf $f(x)$ have cdf of the form $F(x)=H(x)^{\beta}$, where $H(x)$ and $h(x)$ are, respectively, the base cdf and pdf. If $h(x)$ is log-concave, then, for $\beta \geq 1, f(x)$ is log-concave.

The generalized skewed exponential distribution is strongly unimodal. From the discussion in section 2, many reliability properties are also obtained as a consequence of log-concavity.

\subsection{A characterization}

The following characterization is obvious with the cdf form of $F(x)=H(x)^{\beta}$.

Theorem: Let $X_{1}, X_{2}, X_{3}, \ldots, X_{n}$ be independent and identically distributed random variables. Then $X_{i}, i=1,2, \ldots, n$ are generalized skewed exponential random variables if, and only if, $\max _{1 \leq i \leq n}\left(X_{i}\right)$ has a generalized skewed exponential distribution.

This has a physical interpretation as a parallel system with $n$ components, where the system functions only provided at least one of the $n$ components works. If the distributions of the lifetime of the components are independent and identically distributed skewed exponential random variables, then the lifetime distribution of the system has a generalized skewed exponential distribution.

\section{PARAMETER ESTIMATION AND APPLICATION}

\subsection{Parameter estimation}

In this section we derive the score functions for the generalized skewed exponential distribution. The solutions of the score functions are the maximum likelihood estimators. The log-likelihood function can be written as

$\log L(x ; \beta, \alpha, \lambda)=n \log \frac{\alpha \beta(1+\lambda)}{\lambda}+(\beta-1) \sum_{i=1}^{n} \log \left(1-\frac{1}{\lambda}\left((1+\lambda) e^{-\alpha x_{i}}\left(1-e^{-\alpha \lambda x_{i}}\right)\right)\right)-\alpha \sum_{i=1}^{n} x_{i}+\sum_{i=1}^{n} \log \left(1-e^{-\alpha \lambda x_{i}}\right)$ 
Taking the derivatives with respect to $\beta, \alpha, \lambda$, we have the normal equations

$$
\begin{aligned}
& \frac{\partial \log L}{\partial \alpha}=\frac{1}{\alpha n}-\frac{(\beta-1)(1+\lambda)}{\lambda}\left[\frac{e^{-\alpha x_{i}} e^{-\alpha \lambda x_{i}} \lambda x_{i}+\left(1-e^{-\alpha \lambda x_{i}}\right) e^{-x_{i}}}{1-\frac{1+\lambda}{\lambda} e^{-\alpha x_{i}}\left(1-e^{-\alpha \lambda x_{i}}\right)}\right]-\sum_{i=1}^{n} x_{i}+\sum_{i=1}^{n} \frac{\lambda x_{i} e^{-\alpha \lambda x_{i}}}{1-e^{-\alpha \lambda x_{i}}} \\
& \frac{\partial \log L}{\partial \beta}=\frac{n \lambda}{\alpha \beta(1+\lambda)} \frac{\alpha(1+\lambda)}{\lambda}+\sum_{i=1}^{n} \log \left[1-\frac{1}{\lambda}\left((1+\lambda) e^{-\alpha x_{i}}\left(1-e^{-\alpha \lambda x_{i}}\right)\right)\right] \\
& \frac{\partial \log L}{\partial \lambda}=-\frac{n}{\alpha \beta \lambda(1+\lambda)}+(\beta-1) \sum_{i=1}^{n} \frac{e^{-\alpha x_{i}}\left(\frac{1}{\lambda^{2}}\left(1-e^{-\alpha \lambda x_{i}}\right)-\frac{1+\lambda}{\lambda}\left(\alpha x_{i} e^{-\alpha \lambda x_{i}}\right)\right)}{1-\frac{1}{\lambda}\left((1+\lambda) e^{-\alpha x_{i}}\left(1-e^{-\alpha \lambda x_{i}}\right)\right)}
\end{aligned}
$$

The maximum likelihood estimates are obtained by numerically solving equations (4.2), (4.3) and (4.4) simultaneously. We have adopted an alternative method, by directly finding the parameter values of $(\alpha, \beta, \lambda)$ that globally maximize the log-likelihood function (4.1).

\subsection{Application}

A plot of the Malaysia youth unemployment data for period 1997-2015 (Department of Statistics Malaysia) for ages 16 to 60 is shown in Figure 4.1.

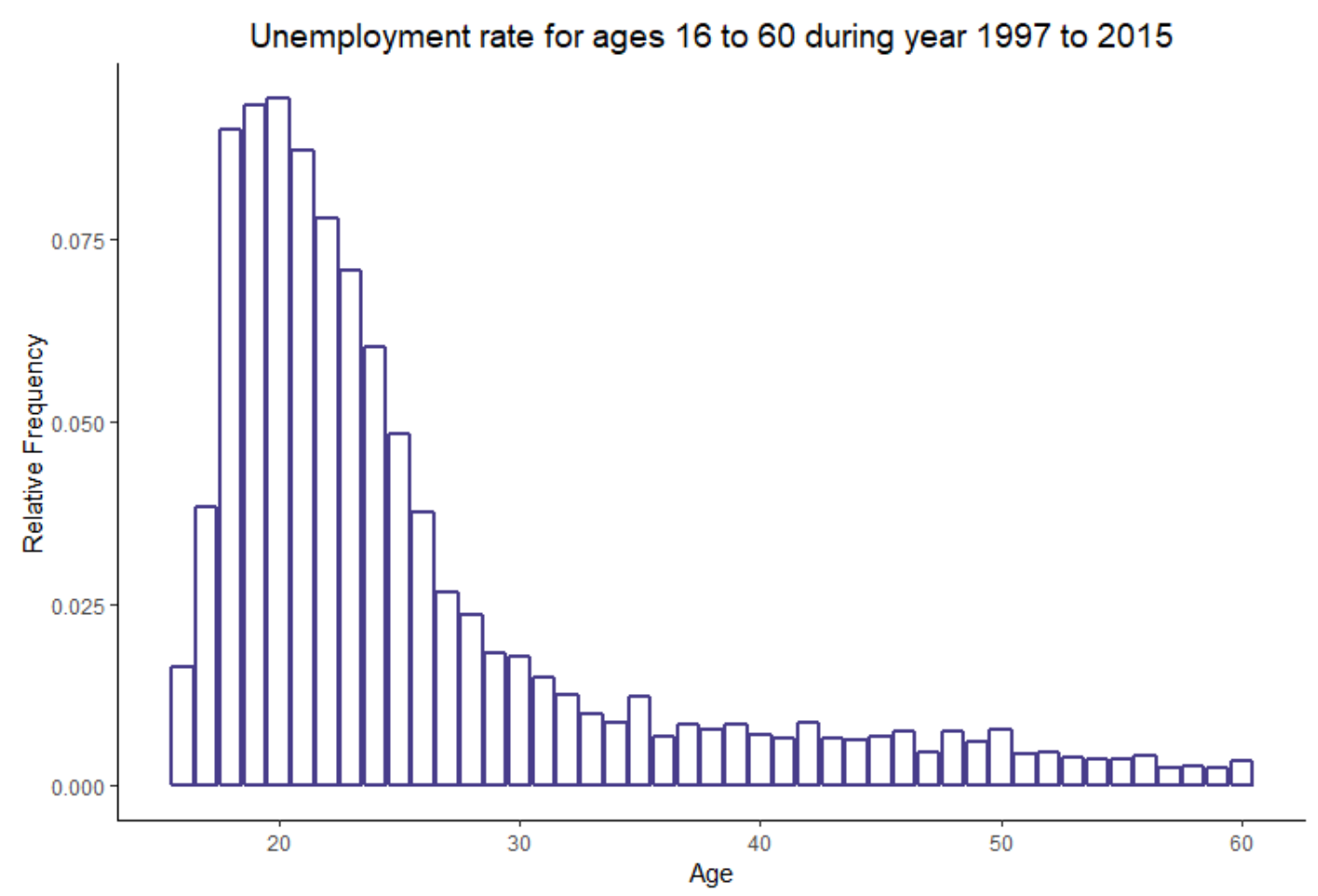

Figure 4.1. Unemployment rate for ages 16 to 60, period 1997-2015 
Table 4.1 tabulates the respective probability density functions for reference purpose. This aggregated data over the period 1997-2015 have been fitted to the Weibull, lognormal, logistic, gamma, skewed exponential and generalized skewed exponential distributions by maximum likelihood estimation. The results are summarized in Table 4.2.

Table 4.2 shows the superior fit of the generalized skewed exponential distribution to the age distribution of the unemployment data as measured by the loglikelihood function and the AIC values.

Figure 4.2 shows the distribution fits for the unemployment data. Although the generalized skewed exponential distribution has fitted the data better than the other models, it is noted that the fit at the mode is only fairly adequate. Further extension or generalization of the distribution will be of interest.

Table 4.1. Probability density function of Weibull, Lognormal, Logistic and Gamma

\begin{tabular}{ll}
\hline Distribution & Probability density function \\
\hline Weibull & $f(x \mid a, b)=\frac{b}{a}\left(\frac{x}{a}\right)^{b-1} e^{-\left(\frac{x}{a}\right)^{b}}, x \geq 0$ \\
Lognormal & $f(x \mid \mu, \sigma)=\frac{1}{x \sigma \sqrt{2 \pi}} \exp \left\{\frac{-(\log x-\mu)^{2}}{2 \sigma^{2}}\right\}, x>0$ \\
Logistic & $f(x \mid \mu, \sigma)=\frac{\exp \left\{\frac{x-\mu}{\sigma}\right\}}{\sigma\left(1+\exp \left[\frac{x-\mu}{\sigma}\right]\right)^{2}}, x \in(-\infty, \infty)$ \\
Gamma & $f(x \mid a, b)=\frac{1}{b^{a} \Gamma(a)} x^{a-1} e^{\frac{-x}{b}}, x \geq 0$ \\
$\begin{array}{l}\text { Skewed } \\
\text { Exponential } \\
\text { Generalized } \\
\text { Skewed } \\
\text { Exponential }\end{array}$ & $\alpha \frac{(1+\lambda)}{\lambda} \exp (-\alpha x)(1-\exp (-\alpha \lambda x))$ \\
\hline
\end{tabular}

Table 4.2. Fits of age distribution of unemployed for ages 16 to 60, period 1997-2015

\begin{tabular}{llll}
\hline Distribution & Parameter estimates & Log-likelihood & AIC \\
\hline Weibull & $\hat{a}=28.9568, \hat{b}=2.7853$ & -29242 & 29246 \\
Lognormal & $\hat{\mu}=3.1987, \hat{\sigma}=0.3055$ & -27571 & 27575 \\
Logistic & $\hat{\mu}=24.0695, \hat{\sigma}=4.6434$ & -28797 & 28801 \\
Gamma & $\hat{a}=9.7795, \hat{b}=2.6391$ & -28075 & 28079 \\
Generalized skewed & $\hat{\beta}=14.4241, \hat{\alpha}=0.1786, \hat{\lambda}=0.05$ & $\mathbf{- 2 7 3 7 7}$ & $\mathbf{2 7 3 8 3}$ \\
exponential & & & \\
Skewed exponential & $\hat{\alpha}=0.0723, \hat{\lambda}=0.1510$ & -34966 & 34970 \\
\hline
\end{tabular}




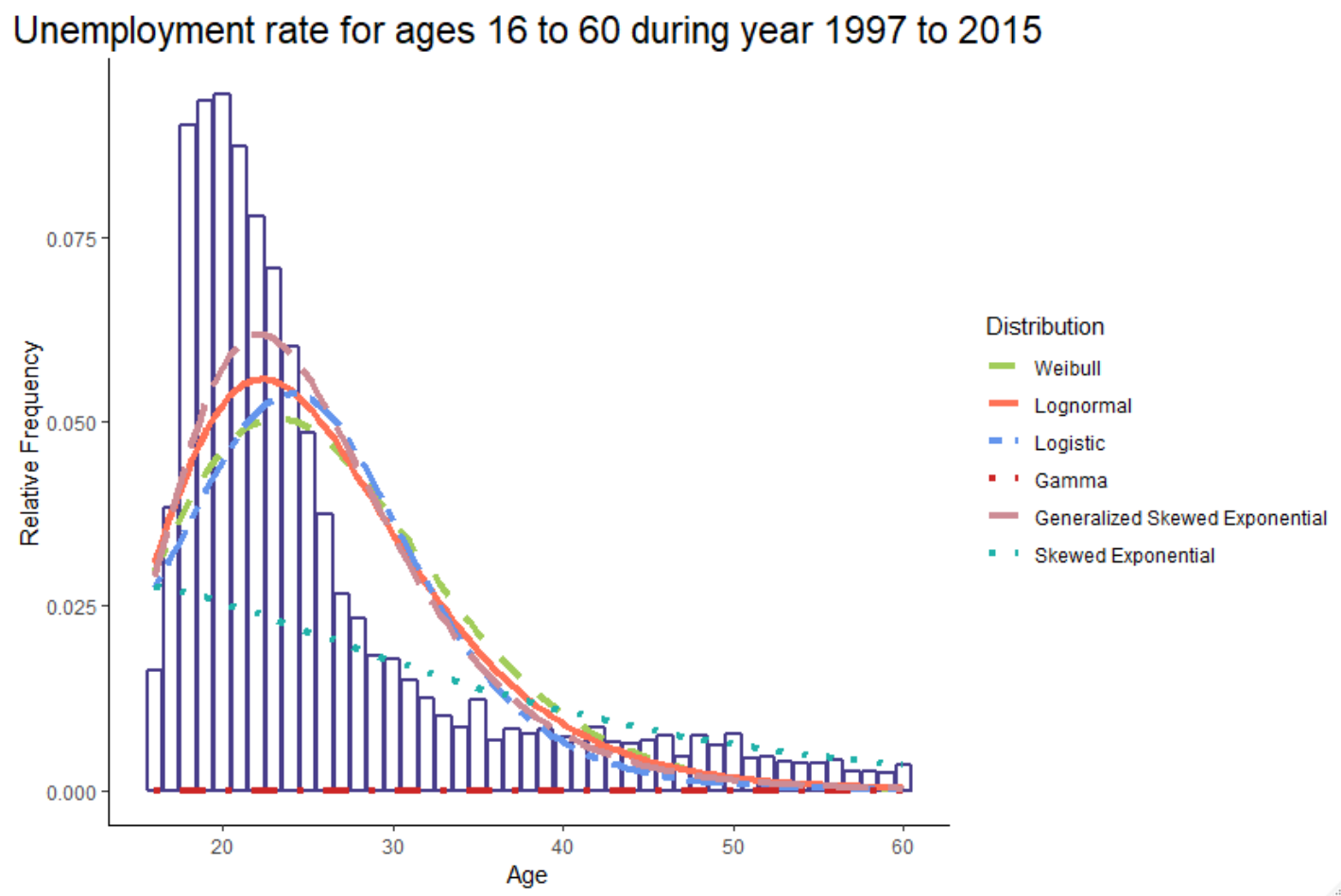

Figure 4.2. Distribution fits for unemployment rate for ages 16 to 60, period 1997-2015

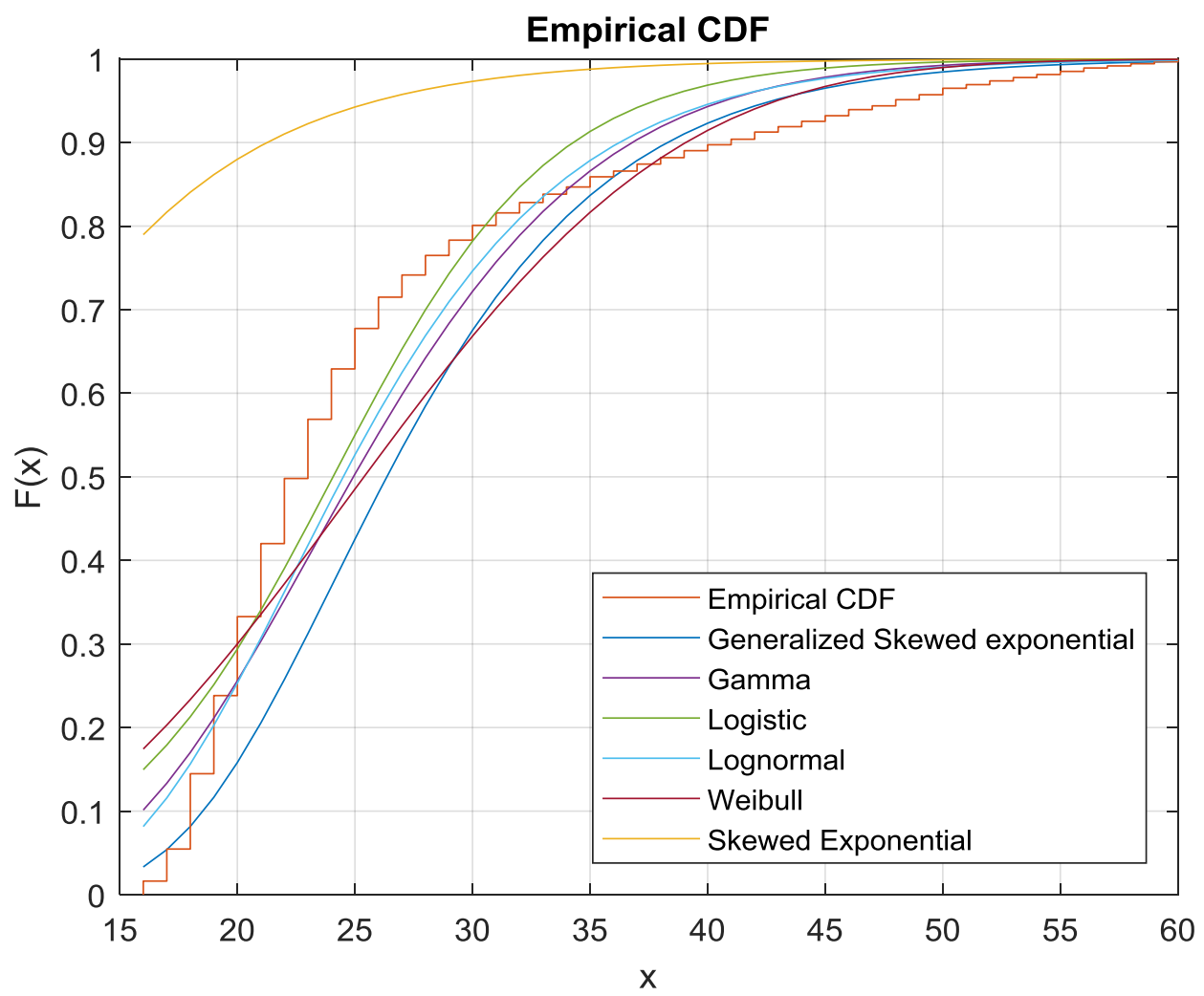

Figure 4.3. Fits of the empirical and theoretical cdf's. 
Table 4.3. KS test statistics and bootstrapped KS critical values for the fitted distributions

\begin{tabular}{cccc}
\hline Distribution & KS test statistic & KS critical value & p-value \\
\hline Weibull & 0.1922 & 0.1976 & 0.592 \\
Lognormal & 0.1560 & 0.1613 & 0.478 \\
Logistic & 0.1628 & 0.1664 & 0.514 \\
Gamma & 0.1757 & 0.1815 & 0.526 \\
Generalized skewed & 0.2603 & 0.2925 & 0.572 \\
exponential & & & 0.000 \\
Skewed exponential & 0.3693 & 0.3547 & \\
\hline
\end{tabular}

We have also considered the goodness-of-fit of the distributions by the Kolmogorov-Smirnoff (KS) test. Figure 4.3 displayed the empirical and theoretical cdf's. Since parameters of the models have been estimated the $\mathrm{p}$-values are determined through simulation. We consider 500 bootstrapped sample size for all fitted distributions. The bootstrapped KS critical values and p-values are presented in Table 4.3. In terms of likelihood function values and AIC (Table 4.2), the generalized skewed exponential distribution provides the best fit while the KS test showed that the Weibull model also provides a good fit.

\section{CONCLUDING REMARKS}

Although it is mathematically straightforward to derive the generalized skewed exponential distribution, the objective of this paper is to determine an empirical model for the unemployed age distribution in Malaysia. In the absence of a stochastic mechanism to explain the age distribution, an empirical model is useful as a summarization and description of this age distribution. The generalized skewed exponential distribution has been shown to fit much better than the well-known logistic, lognormal, Weibull and gamma models. Important properties of the generalized skewed exponential distribution, like logconcavity, have been derived. It is envisaged that the generalized skewed exponential distribution will be useful for statistical analysis in other disciplines.

\section{REFERENCES}

Alzaatreh A, Lee C. and Famoye F. (2013) A new method for generating families of continuous distributions. Metron. 71(1), 63-79.

Azzalini, A. (1985). A class of distributions which includes the normal ones, Scandinavian Journal of Statistics, 12, 171-178.

Bagnoli, M. and Bergstrom, T. (2005). Logconcave probability and its applications. Economic Theory, 26, 445-469

Balabdaoui, F., Rufibach, K. and Wellner, J.A. (2009). Limit distribution theory for maximum likelihood estimation of a log-concave density. Ann. Stat., 37, 1299-1331.

Borzadaran, G. R. M. and Borzadaran, H. A. M. (2011). Log-concavity property for some well-known distributions. Surveys in Mathematics and its Applications, 6, 203-219

Gupta, R.D. and Kundu, D. (1999). Generalized Exponential Distributions. Australian and New Zealand Journal of Statistics, 41, 173-188.

Gupta, R.D. and Kundu, D. (2009). A new class of weighted exponential 
distributions, Statistics, 43(6), 621634.

Jamalizadeh, A. and Lin, T. (2017). A general class of scale-shape mixtures of skew-normal distributions: properties and estimation, Computational Statistics, 32(2), 451-474.

Lee, S.X. and McLachlan, G.J. (2016). Finite mixtures of canonical fundamental skew t- distributions: The unification of the restricted and unrestricted skew t-mixture models, Statistics and Computing, 26, 573589.
Lin T., Wang, W.L. McLachlan, G.J. and Lee, S.X. (2018). Robust mixtures of factor analysis models using the restricted multivariate skew-t distribution, Statistical Modelling, 18(1), 50-72.

Marshall, A.N. and Olkin, I. (1997). A new method for adding a parameter to a family of distributions with applications to the exponential and Weibull families. Biometrika. 84, 641-652

Ochsen, C. (2008). The Distribution of Unemployment by Age and the Unemployment Rate - A Puzzle? Working Paper No. 89, ThünenSeries of Applied Economic Theory, Universität Rostock. 